To appear in PASP (February issue)

\title{
Metal-rich globular clusters of the Galaxy: morphology of the helium-burning "clump," and the determination of relative ages through the " $\Delta V$ " method
}

\author{
M. Catelan \\ Instituto Astronômico e Geofísico, Universidade de São Paulo \\ C.P. 9638, CEP 01065-970 São Paulo, SP \\ Brasil \\ e-mail: marcio@vax.iagusp.usp.br \\ and \\ J. A. de Freitas Pacheco \\ Observatoire de la Côte d'Azur \\ B.P. 229, 06304 Nice Cedex 04 \\ France \\ e-mail: pacheco@rameau.obs-nice.fr
}

\begin{abstract}
Morphological aspects of the $V, B-V$ diagram of metal-rich globular clusters are analyzed, on the basis of stellar evolution models for horizontal-branch (HB) and red giant branch stars. These have been incorporated into detailed synthetic HB models, the influence of differential reddening upon which is also discussed. The synthetic HB models are found to be very clumpy in the colour-magnitude diagram, though more extended structures occasionally result due to the effect of evolution away from the zero-age HB. In particular, "sloped" clumps may be naturally expected for sufficiently high helium abundances. Differential reddening is found to have a smaller influence than evolution itself upon the morphology of the clump. The synthetic HB models are also used to study the age difference between metal-rich clusters on the basis of the " $\Delta V$ " method. We emphasize the importance of knowing in advance the relative abundances of helium and metals in order to estimate this age difference quantitatively. We present chemical evolution models that serve to illustrate the effect, and show that, for two clusters of identical $[\mathrm{Fe} / \mathrm{H}]$ and $\Delta V$, the one enriched predominantly by ejecta from supernovae type II explosions will appear younger than the other which also suffered enrichment from supernovae type Ia ejecta. The implied locations of the red giant branch feature known as the "bump" are also discussed on the basis of recent observational and theoretical results. Our corresponding predictions should be checked against accurate observational data obtained with the Hubble Space Telescope.
\end{abstract}


Subject headings: Galaxy: Globular Clusters: General — Galaxy: Evolution — Stars: Evolution — Stars: Giants — Stars: Horizontal-Branch — Stars: Population II. 


\section{Introduction}

Recently, CCD photometry of metal-rich Galactic globular clusters (MRGCs), such as NGC 6553 (Ortolani, Barbuy \& Bica 1990, hereinafter referred to as OBB90) and Terzan 1 (Ortolani, Bica \& Barbuy 1993), has been obtained with the purpose of revealing the morphology of the main branches in their colour-magnitude diagrams (CMDs), and, when possible, to investigate their ages and establish a chronology for the formation of the different structures (halo, disk, bulge) that comprise the present-day Galaxy. Many interesting results emerged from these investigations, among which the suggestion of a possible "tilted" helium-burning "clump" or horizontal branch (HB) in the $V, B-V$ CMDs of MRGCs (OBB90; see also Armandroff 1988). However, the significance of this result was not well established in these studies, and indeed some controversy still exists as to its reality and extension. From the theoretical point of view, in particular, the present authors are aware of no investigation aiming at a more detailed analysis of the allowed clump morphologies in MRGCs on the basis of available evolutionary tracks, except for the study of Dorman, VandenBerg \& Laskarides (1989) on 47 Tuc. In addition, in spite of the remarkable observational efforts by the above and other authors, no conclusive results could be obtained about the ages of the studied objects - especially those located in the Galactic bulge.

Establishing a reliable chronology for the formation of the Galactic subsystems will be possible in the near future. Indeed, several observational projects on many MRGCs that are presently being carried out by different groups employing the Hubble Space Telescope (HST) will soon be providing us with CMDs of unprecedented accuracy, and standard age-determination techniques will be able to shed light on the problem of the relative ages of the halo, bulge, and disk.

In the present study, we carry out a theoretical investigation of the morphology of the HB clump in MRGCs on the basis of the detailed synthetic HB models which are described in Sect. 2. We demonstrate, in Sect. 3, that the production of synthetic models with tilted clumps is not a general consequence of evolution away from the zero-age HB (ZAHB), even though it can be achieved for models with sufficiently high helium abundances, and/or in fields where differential reddening is very large. On the basis of the " $\Delta V$ " method and different chemical evolution scenarios for the MRGCs of given $\Delta V$ (the magnitude difference between the clump and the turnoff), the theoretical problem of the determination of the age difference between two MRGCs is investigated in Sect. 4. We show that knowledge of the relative abundances of helium and metals is essential in order to determine the difference in age between any two MRGCs in a reliable way. In particular, we emphasize that this may be a most important point when comparing bulge and disk globulars of similar $[\mathrm{Fe} / \mathrm{H}]$, since their chemical evolution histories may have been different. For this reason, a strong effort must be made to determine the abundances of helium and the $\alpha$-capture elements in these clusters from the observations. In Sect. 5, we evaluate the theoretically-expected location of the red giant branch (RGB) feature known as the "bump" with respect to the clump. Finally, in Sect. 6 we summarize our results and provide some additional discussion. 


\section{Synthetic models of the HB clump}

Catelan (1993) has computed synthetic HB models for low metallicities in the theoretical plane. In the present work, we extend his calculations by including published evolutionary tracks for higher metallicities. The evolutionary masses considered match the HB morphology that is usually observed in metal-rich objects, where the stars lie entirely to the red of the instability strip (Armandroff 1988).

The incorporated evolutionary tracks for HB stars include the models computed by Sweigart (1987, hereinafter SW87) for $\left(Y_{\mathrm{MS}}, Z\right)=(0.25,0.01)$ and $\left(Y_{\mathrm{MS}}, Z\right)=(0.30,0.01)$, as well as those by Castellani, Chieffi \& Pulone (1991, hereinafter CCP91) - based upon improved input physics - for $\left(Y_{\mathrm{MS}}, Z\right)=(0.23,0.006)$ and $\left(Y_{\mathrm{MS}}, Z\right)=(0.23,0.02)$. $Y_{\mathrm{MS}}$ represents, as usual, the helium abundance prior to the main-sequence phase.

To perform the transposition of the models from the theoretical to the observational $\left(M_{V}, B-V\right)$ plane, we employ the conversions from VandenBerg 1992 (hereafter VdB92), which are actually a smooth match of the VandenBerg \& Bell (1985) atmospheres to those of Bell \& Gustafsson (1978) and of Kurucz (1979). His published tables are restricted to the range $3500 \mathrm{~K}<T_{\text {eff }}<8000 \mathrm{~K}$ - which turns out to be adequate for our present purposes. It is important to note that the specific procedures adopted by VdB92 in obtaining these conversions do not introduce significant uncertainties into our results, since both the colour transformations and bolometric corrections $B C_{V}$ of $\mathrm{VdB} 92$, in the temperature and gravity range of our models (cf. Figs. $1-4$ ), coincide closely with those from Bell \& Gustafsson 1978. As recommended by VdB92, Hill's (1982) Hermite interpolation algorithm has been employed for interpolation with respect to $T_{\text {eff }}$ and $\log g$, and linear interpolation with respect to $[\mathrm{M} / \mathrm{H}]$.

Observational errors in the photometry have also been included in the present models. We adopt $\sigma_{V}=0.035 \mathrm{mag}$ and $\sigma_{B-V}=0.046 \mathrm{mag}$ at the level of the HB, as estimated from the study of OBB90 for the MRGC NGC 6553.

We plan to extend the present calculations in the future by including the transformations to the infrared planes, employing the prescriptions of Bell \& Gustafsson (1989) and Bell (1992).

\section{Expected morphology of the metal-rich clumps}

Figs. 1, 2 and 3 demonstrate that the production of tilted HB clumps is not generally expected, but rather a matter of fine-tuning of evolutionary parameters of HB evolution. Figs. 1a and b demonstrate that the CCP91 evolutionary tracks do not produce significantly tilted synthetic HB models, irrespective of $Z$. This is probably related to the low helium abundance employed in the CCP91 calculations: the SW87 tracks for $\left(Y_{\mathrm{MS}}, Z\right)=(0.25,0.01)$ do not lead to

sloped models either (cf. Figs. 1c and 2a), as opposed to the $\left(Y_{\mathrm{MS}}, Z\right)=(0.30,0.01)$ combination (cf. Fig. 3a). Indeed, in the latter case, for $\left\langle M_{\mathrm{HB}}\right\rangle \simeq 0.66-0.68 M_{\odot}$, tilted models are clearly 
obtained.

Inspection of Figs. 7 through 9 in SW87 does show that, for stars in this mass range, the "blueward loops" (which characterize the HB evolutionary tracks for stars in which the H-burning shell is a major contributor to the total luminosity; cf. Iben \& Rood 1970) present a trend of increasing slope $\mathrm{d} \log L / \mathrm{d} \log T_{\text {eff }}$ with increasing $Y$. A similar conclusion may be drawn from close inspection of the diagrams published by Seidel, Demarque \& Weinberg (1987), but the effect is especially clear in Figs. 1 through 5 of Sweigart \& Gross (1976), where the computations were extended up to $Y=0.40$, and in the diagrams presented by Dorman et al. (1989) in their detailed analysis of the HB morphology in 47 Tuc. Sweigart \& Gross interpret this increase in slope of the blueward loops with increasing $Y$ as being a direct consequence of the increasing rate of shell H-burning, which makes the H-shell advance outward more rapidly through the stellar envelope. Thus, we expect that a very high helium abundance for the bulge stars, as might be present if a large contribution to the chemical enrichment from type II supernovae took place (cf. Sect. 4 below), would lead more naturally to sloped HBs. Unfortunately, we cannot present synthetic models for $Y_{\mathrm{MS}}>0.30$, since the required extensive grids of $\mathrm{HB}$ evolutionary tracks are presently lacking.

This result, in fact, is not new: Dorman et al. (1989) have suggested employing the slope of the red clump as a diagnostic of the helium abundance in GCs with red HBs, and from this have estimated that $Y$ in 47 Tuc probably lies midway between 0.20 and 0.30 .

The reader will find instructive to compare the synthetic model in Fig. 1a with the observed CMDs for 47 Tuc that have been published by Hesser et al. (1987) and Carney, Storm \& Williams (1993). The general agreement appears quite satisfactory. Note that the final evolution to the asymptotic GB, which is clear in the observed CMD, is present in the CCP91 tracks, but lacking in the SW87 case. Note as well that the slope of the lower envelope of the HB distribution, which is present in the synthetic model but not in $Z A H B$ sequences, serves as a major caution against using ZAHB models in applications to clusters with red HBs (Dorman et al. 1989).

The presence of a tilt in the $M_{V}, B-V$ diagram in the cases where there was none in the corresponding theoretical diagram is a consequence of the blanketing contained in the VdB92 transformations, which affects stars in a different way as a function of their temperatures along the HB (compare Figs. 3a and 4). Indeed, inspection of Fig. 7 of VdB92 shows that, in the relevant temperature range, $3 \lesssim \mathrm{d} B C_{V} / \mathrm{d} \log T_{\text {eff }} \lesssim 6$. If such a trend is not counterbalanced by an opposite slope of the blueward loop in the $M_{\text {bol }}-\log T_{\text {eff }}$ diagram (cf. Fig. 4), a tilted model will clearly result - which is the reason for the peculiar slope of the model in Fig. 3a. This effect is also clear in Dorman's (1992) Figs. 10 and 11.

Differential reddening is generally expected to affect the CMD morphology of most MRGCs, since they generally lie very close to the Galactic plane. According to OBB90, for instance, in the specific case of NGC 6553 this effect may account for a vertical dispersion of $\Delta V \simeq 0.18$ mag and a horizontal dispersion of $\Delta(B-V) \simeq 0.06 \mathrm{mag}$ in the cluster's CMD. Thus, differential reddening 
works in the sense of bringing the clumpy models (cf. Figs. 1 and 2a) into tilted structures.

This is confirmed by our synthetic HB models. The effect is illustrated in Figs. 2b-d and 3b. In the former panels, increasing amounts of (uniform) differential reddening are assumed in the model construction, in comparison with the unreddened diagram depicted in Fig. 2a. Similarly, in Fig. 3b the unreddened synthetic HB displayed in Fig. 3a is changed in order to simulate the effects of the differential reddening that OBB90 estimate to be present in the field of NGC 6553 [i.e., $\Delta E(B-V) \simeq 0.06 \mathrm{mag}$ ]. Differential "reddening," in these simulations, is defined so that the mean reddening amounts to zero.

It is clear, from Figs. $2 \mathrm{a}-\mathrm{b}$ and $3 \mathrm{a}-\mathrm{b}$, that a differential reddening as small as $\Delta E(B-V)=0.06 \mathrm{mag}$ cannot cause a CMD dispersion as large as the one originating from the evolution away from the ZAHB itself. Thus, unless differential reddening in the field of a MRGC is very large (Figs. 2c-d), it seems unlikely that sloped HBs can be produced on this basis alone.

Thus, in the case of the MRGC NGC 6539, it is very likely that the primary cause for the sloping HB is indeed the large amount of differential reddening that is present in the field of this cluster, as Fig. 7 in Armandroff 1988 convincingly demonstrates. In the case of NGC 6553, on the other hand, this possibility may appear somewhat less appealing.

To be sure, the above conclusions rely on the adequacy of the VdB92 blanketings for cool stars. Dorman (1994) finds that the VdB92 transformations can reproduce the observational data quite satisfactorily. At any rate, inclusion of the Kurucz $(1991,1992)$ and Bell \& Tripicco (1995) blanketings should prove of interest in the present context. Similarly, a more extended and homogeneous set of updated evolutionary tracks for metal-rich compositions would definitely prove very useful. Also, the computation of synthetic models in the infrared would be of great interest for a variety of reasons: for instance, while MRGCs are heavily obscured at optical wavelengths, in the infrared region they are less subject to interstellar extinction (e.g., Guarnieri et al. 1995), which also renders the effect of differential reddening a less important problem (Davidge \& Simons 1994). Transforming models similar to the present ones to the infrared plane on the basis of the model atmospheres by Bell \& Gustafsson (1989) and Bell (1992) is a necessary future step in the investigation of the expected morphology of HB clumps in MRGCs. We emphasize the need of synthetic calculations employing extensive grids (in $Y, Z$, and $M_{\mathrm{HB}}$ ) of $\mathrm{HB}$ evolutionary tracks to perform more detailed comparisons with the observed CMDs, since neither ZAHB sequences nor individual evolutionary tracks are expected to be able to adequately represent the observed structures (Dorman et al. 1989).

Before closing the present section, we believe it may be of interest to note that ZAHB sequences do become themselves tilted structures at sufficiently high values of $M_{\mathrm{HB}}$ (e.g., Seidel et al. 1987). This may also contribute to the possible tilting of HB clumps. For instance, Fig. 3 in Tripicco, Dorman \& Bell 1993 shows that, for $Y_{\mathrm{MS}}=0.27$ and $Z=0.02$, the sign of $\mathrm{d} \log L / \mathrm{d} \log T_{\text {eff }}$ for the ZAHB becomes positive for $M_{\mathrm{HB}} \gtrsim 0.9 M_{\odot}$. Our models have all been 
built for significantly lower masses. However, this would demand ages $\lesssim 8.9$ Gyr for a (rather modest) overall mass loss on the RGB of $\Delta M \sim 0.1 M_{\odot}$. Thus, this only becomes a realistic possibility for sufficiently young MRGCs.

\section{The age difference between MRGCs from the $\Delta V$ method}

\subsection{Theoretical approach}

There is not a sufficiently accurate method to estimate absolute ages of MRGCs. For instance, the $\Delta V$ method cannot be directly employed due to the many uncertainties involved in, e.g., the helium abundance and the HB magnitude scale. Even relative ages may be difficult to determine. The $\Delta(B-V)_{\mathrm{TO}, \mathrm{RGB}}$ method, while very useful in applications to metal-poor objects, cannot be reliably applied to MRGCs, since age and $[\mathrm{Fe} / \mathrm{H}]$ differences may be hard to disentangle at the metal-rich end (VandenBerg, Bolte \& Stetson 1990; Bolte 1992). This, unfortunately, is a most important point in what regards MRGCs, since $[\mathrm{Fe} / \mathrm{H}]$ values for these objects are often known only with large uncertainties (Bolte 1992).

The following discussion is aimed at highlighting the important ingredients that even a differential age determination for MRGCs based upon the $\Delta V$ method should include.

From Eq. (1) in Renzini 1991 and the analysis of Chieffi, Straniero \& Salaris (1991; see also Salaris, Chieffi \& Straniero 1993), one derives

$$
\Delta \log t_{9}=0.37\left[\Delta M_{V}^{\mathrm{HB}}+\Delta\left(\Delta V_{\mathrm{HB}}^{\mathrm{TO}}\right)\right]-0.43 \Delta Y_{\mathrm{MS}}-0.13 \Delta[\mathrm{Fe} / \mathrm{H}]-0.13 \Delta \log (0.579 f+0.421),
$$

where $\Delta V_{\mathrm{HB}}^{\mathrm{TO}}$ is the difference in magnitude between the turn-off region and the $\mathrm{HB}$, and $f=10^{[\alpha / \mathrm{Fe}]}$. We assume this relation to be approximately valid up to near-solar metallicities. We see that helium and the $\alpha$-elements play a rôle. To be noted, in particular, is that $M_{V}^{\mathrm{HB}}$ depends on $Y_{\mathrm{MS}},[\mathrm{Fe} / \mathrm{H}]$, and $f$ as well. These abundances are not directly known for MRGCs from the observations, and one must often resort to theoretical scenarios to estimate them (e.g., Renzini \& Greggio 1990). From the following discussion, it will become clear that observational determinations of these quantities are urgently needed - and in fact at least the $[\alpha / \mathrm{Fe}]$ ratio may be obtained directly from spectroscopic measurements.

The time evolution of the abundance of a given element is not well predicted yet. Among the reasons for this is the controversy that still exists about the enrichment timescale for metal-poor stars. While the onset of type Ia supernovae (SN Ia) may require a relatively long scale $\tau \gtrsim 10^{9}$ yr (van den Bergh 1991), the evidence from kinematical studies of field stars and the analysis of CMDs of GCs remains somewhat controversial. Since addressing these subjects is beyond the scope of our article, we refer the reader to the following papers for additional information and references: Beers \& Sommer-Larsen 1995 (field-star kinematics); Catelan \& de Freitas Pacheco 
1995 (ages of the halo GCs); Marquez \& Schuster 1994 (ages of field stars). The reviews by Majewski (1993) and Matteucci (1995) address these points at some length.

These uncertainties notwithstanding, element ratios as a function of metallicity can be computed, following the method by de Freitas Pacheco (1993) and Barbuy, de Freitas Pacheco \& Castro (1994). Such a procedure requires the knowledge of the relative yields due to massive stars (SN type II and maybe type Ib) and to SN Ia. The relative number of events of different supernovae types is also necessary in order to compute these chemical evolution models.

For the present purposes, we have considered three distinct scenarios which should serve to illustrate reasonably well the associated uncertainties in applications of the $\Delta V$ method. In the first (Case 1), we assume that in the metallicity range $-3.0<[\mathrm{Fe} / \mathrm{H}]<+0.5$ the chemical enrichment was produced by SN II only. In the second (Case 2), we consider that at $[\mathrm{Fe} / \mathrm{H}]=-1.0$ occurs the onset of SN Ia, with an increasing contribution to the yields until a maximum of $10 \%$ in the relative number of events. In the last scenario (Case 3), we examine a higher contribution of SN Ia to the chemical enrichment, amounting to about $40 \%$ in the relative number at $[\mathrm{Fe} / \mathrm{H}]=+0.5$.

The yields from massive stars were computed by averaging over the initial mass function (Salpeter's 1955 law) the results by Arnett (1990). For SN Ia, we used the yields of model W7 by Nomoto, Thielemann \& Yokoi (1984). The initial He abundance (cosmological value) was taken to be equal to 0.075 by number. Fig. 5a shows the He abundance by mass as a function of the metallicity $[\mathrm{Fe} / \mathrm{H}]$. Fig. $5 \mathrm{~b}$ shows the $[\mathrm{O} / \mathrm{Fe}]$ ratio (representative of the abundance of the $\alpha$-elements) versus $[\mathrm{Fe} / \mathrm{H}]$ for the three considered scenarios.

Let us examine the age difference between two given GCs. For MRGC1, we assume Case 1 models to be valid, and adopt $[\mathrm{Fe} / \mathrm{H}]=-0.71$. Hence, $Y_{\mathrm{MS}} \simeq 0.266$ and $[\alpha / \mathrm{Fe}] \simeq+0.35$ for this object (cf. Fig. 5a). For MRGC2, in turn, we investigate the implications of all three different chemical evolution scenarios, assuming $[\mathrm{Fe} / \mathrm{H}]=-0.2$. The resulting abundance parameters are displayed in Table 1.

According to Eq. (1), an estimate of the difference in absolute magnitude between the HBs of the two MRGCs is also necessary. This was accomplished as follows. On the basis of the discussion in the next section, we adopt $\Delta M_{V}^{\mathrm{HB}} / \Delta \log Z \approx(1.33-0.98) /(-1.7+2.22) \simeq 0.67$. Following our synthetic clump models based upon the SW87 tracks for $Y_{\mathrm{MS}}=0.25$ and $Y_{\mathrm{MS}}=0.30$, we take $\Delta M_{V}^{\mathrm{HB}} / \Delta Y_{\mathrm{MS}} \approx(0.6-0.8) /(0.30-0.25)=-4$. The resulting $\Delta M_{V}^{\mathrm{HB}}$ values (MRGC1 minus MRGC2) are also displayed in Table 1, as are the inferred age differences.

[It is to be noted, from these estimates, that a ratio of $\approx-6$ is found between the $Y_{\mathrm{MS}}$ and $\log Z$ dependencies of $M_{V}^{\mathrm{HB}}$ from the models. On the other hand, from Fig. 1 in Demarque \& Lee 1992 a ratio of $\approx-10$ is estimated instead. Since the latter models are also based upon older input physics in comparison with the CCP91 study, this clearly illustrates the effect of improved opacities upon the model predictions for high-metallicity stars.]

In Table 1, a value of $\Delta V=3.6$ has been assumed for MRGC1, which is a quite normal value 
found among old halo GCs. For MRGC2, two different values have been considered, $\Delta V=3.3$ and 3.6. In fact, Fig. 5 and Eq. (1) may easily be employed for the determination of age differences for arbitrary $[\mathrm{Fe} / \mathrm{H}]$ and $\Delta V$.

We see, from an analysis of Table 1, that the different assumptions about the chemical evolution of the metal-rich system affect the derived age differences in a significant way. Note, in particular, that for a given $\Delta V$ value, MRGCs which have been formed from gas enriched primarily by SN II explosions appear to be somewhat younger than those which have also been subject to SN Ia ejecta (Cases 2 and 3). This may be a most important point when comparing ages of disk and bulge globulars of similar $[\mathrm{Fe} / \mathrm{H}]$.

To illustrate the point, we call the reader's attention to the following quantitative estimates. MRGC1 will be considered older by $15 \%$ than MRGC2 for $\Delta V($ MRGC2) $=3.60,3.45$, and 3.33 in Cases 1, 2, and 3, respectively. Similarly, coevality between the two objects will be reached for $\Delta V(\mathrm{MRGC2})=3.77,3.61,3.49$ in these three Cases, respectively. As before, $[\mathrm{Fe} / \mathrm{H}]=-0.2$ has been assumed for MRGC2, and $[\mathrm{Fe} / \mathrm{H}]=-0.71$ and $\Delta V=3.6$ for MRGC1.

The above conclusions would change if, as pointed out at the end of Sect. 3, the clump structure is populated by stars more massive than $\approx 1.0 M_{\odot}$. In this case, the HB of MRGC2 (which has a higher $[\mathrm{Fe} / \mathrm{H}]$ value) could be brighter by $\approx 0.35$ mag than assumed herein (Castellani, Chieffi \& Straniero 1992). As a consequence, as is apparent from Eq. (1), the age difference would increase (i.e., MRGC2 would be younger), in agreement with the requirement of smaller ages to account for the higher masses that would be reached at the tip of the RGB.

\subsection{On applications of the method to real clusters}

In the first place, one should note that the above results have been inferred on the basis of a theoretical analysis of the variation of the HB magnitude with the metallicity and helium abundance. Observational estimates of the magnitudes of the core helium-burning stars are usually obtained for more metal-poor GCs with populated instability strips (see, e.g., Sandage \& Cacciari 1990; Sandage 1993; Carney, Storm \& Jones 1992; for recent discussions and references), which is obviously not the case here. Moreover, applying relations obtained for the RR Lyrae level to objects lying to the blue or to the red of the instability strip is not a straightforward procedure: theoretically-estimated corrections are needed with the purpose of roughly obtaining the magnitude of the clump of red HB stars from the estimated magnitudes of the (often missing) RR Lyrae variables (cf. Catelan 1992, 1993 and references therein). For this reason, a comparison with relations obtained for samples of field RR Lyrae stars which do contain a few metal-rich variables (e.g., Skillen et al. 1993) should also be made with caution. In addition, much controversy still exists in what concerns the slope of the $M_{V}^{\mathrm{HB}}-[\mathrm{Fe} / \mathrm{H}]$ relation. This question has been addressed quite recently by several authors (e.g., Carney et al. 1992; Catelan 1992; Fernley 1993; Sandage 1993), without a general consensus having been found. 
Are the chemical abundances predicted by the above chemical evolution models representative of real GCs? As to the model parameters for MRGC1, we note that they may characterize rather satisfactorily the well-studied GC 47 Tuc. This cluster, according to Zinn (1985), has $[\mathrm{Fe} / \mathrm{H}]=-0.71$. The helium abundance implied by our Case 1 models is not incompatible with estimates based upon the " $R$-method" (Buzzoni et al. 1983; Caputo, Martinez Roger \& Paez 1987; Hesser et al. 1987; CCP91), while the abundances of the $\alpha$-elements in these models are in reasonable agreement with the values estimated by Brown, Wallerstein \& Oke (1990) and Brown \& Wallerstein (1992). For this cluster, analysis of the CMD gives $\Delta V=3.6 \pm 0.1$ (Hesser et al. 1987), which is the value that we have adopted for MRGC1 in the above analysis.

As to the model parameters for MRGC2, we note that Zinn (1985) lists $[\mathrm{Fe} / \mathrm{H}] \simeq-0.29$ for the MRGC NGC 6553, for which $\Delta V=3.3$ (OBB90) or $\Delta V=3.6$ (Ortolani 1994, based on analysis of improved HST images). Thus, the corresponding models may be representative of this cluster. As already emphasized, however, the actual abundances of the $\alpha$-elements and helium in the cluster stars are, unfortunately, still unknown in detail. Some insight may be gained from the available estimates for field stars. For stars in the bulge, in particular, Renzini (1994) has recently estimated, on the basis of the $R$-method, a very high helium abundance: $Y \approx 0.30-0.35$. Minniti (1995a), on the other hand, obtains a somewhat lower helium abundance for these stars, $Y=0.28 \pm 0.02$. McWilliam \& Rich (1994) find evidence that the field stars in the bulge are overabundant in the $\alpha$-elements, although their element ratios are not clearly compatible with the expected enrichment patterns for SN II explosions. Also, very recently Idiart, de Freitas Pacheco \& Costa (1995) obtained an integrated spectrum for the bulge in the direction of Baade's Window. Their analysis of metallicity indicators like $M g_{2}$ and $\langle F e\rangle$ points to an average metallicity $[\mathrm{Fe} / \mathrm{H}]=0.0$, and an average ratio $[\mathrm{Mg} / \mathrm{Fe}]=+0.4$. Comparison of these results with the model predictions for the bulge (Fig. 5) would appear to favor Case 1. Case 2 would not be ruled out either. Case 3, on the other hand, would appear as an extreme possibility in applications to MRGCs that do in fact belong to the Galactic bulge, since it implies oxygen abundances at high metallicities which are similar to those obtained by Ratag et al. (1992) for the planetary nebulae located in, but not necessarily belonging to, the Galactic bulge. In this regard, it may be of interest as well to remark that very recently Terndrup \& Walker (1994) have studied in detail the properties of NGC 6522 (located in Baade's Window, at $\simeq 1.5 \mathrm{kpc}$ from the Galactic center), finding this to be a metal-poor, blue-HB cluster — similar to the halo globulars located inside the solar circle. In fact, this leads us to caution that the general correspondence between field and GC stars which are located in a certain (limited) volume of space (in this case, the Galactic bulge) must be established in more detail, employing (among others) the tools from spectroscopic analysis.

Equally important, in this regard, is a precise identification of those individual clusters that belong to the bulge and to the disk. Since these two Galactic subsystems may have had different chemical enrichment histories, it is clearly of the utmost importance to know the population to which the cluster is associated in order to make a quantitative estimate of its relative age in 
comparison with a reference object — 47 Tuc, for example. According to Zinn (1990, 1991), an individual "bulge system of GCs" probably does not exist. According to the recent analysis of a more extended dataset by Minniti (1995b), on the other hand, several MRGCs may indeed belong to the bulge. Our results show that special care should be taken in comparing the ages of these objects with those for other clusters lying outside the Galactic bulge, especially in what regards the assumed chemical composition.

\section{On the expected location of the RGB bump}

In the present section, we present a short compilation and discussion of recent theoretical and observational results for the RGB bump in GCs which will hopefully prove useful in future comparisons with more accurate observational data for the more metal-rich objects. The basic motivation for our analysis are the suggestions by OBB90, Ortolani et al. (1992), and Demarque \& Lee (1992) that the tilted HB clumps in some MRGCs might be due to the proximity of the RGB bump.

The RGB bump occurs as an RGB star has a chemical composition profile discontinuity, resulting from the previous inward penetration of its surface convection layer, suddenly reached by the H-burning shell. When this happens, the luminosity of the star drops, and it appears to hesitate momentarily prior to continuing its journey to the tip of the RGB. As a result, a small, but detectable (Iben 1968; King, Da Costa \& Demarque 1985; Castellani, Chieffi \& Norci 1989; Fusi Pecci et al. 1990, hereinafter FFCRB90; Bono \& Castellani 1992) agglomeration of stars is expected in the regions of the HR and CM diagrams where this phenomenon takes place.

A comprehensive investigation of the occurrence of these RGB bumps in Galactic globulars has been presented by FFCRB90. In this paper, the authors have detected the bump in eleven Galactic globulars with metallicities in the range $-2.15 \leq[\mathrm{Fe} / \mathrm{H}] \leq-0.71$; the only previously reported detection had been for 47 Tuc (King et al. 1985). FFCRB90 attempt to calibrate the $M_{V}^{\mathrm{HB}}-[\mathrm{Fe} / \mathrm{H}]$ relation on the basis of the magnitude difference between the $\mathrm{HB}$ and the detected bump. They find, however, zero-point problems in comparison with independent calibrations of this relation, which demands an offset of the theoretical bump predictions by some +0.4 mag in $M_{V}$ - i.e., predicted bump locations are too bright in comparison with the observations. Catelan (1992) calls attention toward a secondary problem involving the metallicity dependence of their results.

While revised $M_{V}^{\mathrm{HB}}-[\mathrm{Fe} / \mathrm{H}]$ relations have indeed implied somewhat brighter RR Lyrae variables (see Catelan, de Freitas Pacheco \& Horvath 1995 for a review and discussion of the evolutionary implications of this result) than assumed by FFCRB90 in their analysis, a significant zero-point offset is still present.

King et al. (1985) have argued that overshooting at the base of the convective envelope is a likely explanation for this discrepancy, which they as well had previously found. Similarly, Alongi 
et al. (1991) and Bono \& Castellani (1992) note that existing bump models are based on the assumption that there is a sharp discontinuity in the chemical composition profile at the bottom of the convective layer, whereas in the real case a partially mixed region is to be expected. From Table 1 in Alongi et al. 1991 and Fig. 2 in Bono \& Castellani 1992, one may easily conclude that, upon relaxation of this simplifying hypothesis, the bump can be made fainter by a few tenths of a magnitude, while remaining a firm theoretical prediction. A small contribution may also come from the effect of mass loss on the RGB (Castellani \& Castellani 1993).

Improved opacities (FFCRB90) cannot alter the predicted bump magnitudes significantly for low metallicities (Alongi et al. 1991; see also Ferraro 1992), although they may become important for higher metallicities (see below). The influence of $\alpha$-elements, which may be important for the bump magnitudes (Ferraro 1992), has been accounted for in the present paper by computing $Z$ values from the Chieffi et al. (1991) expression.

More realistic, extensive models lacking, however, we are forced to restrict the present analysis to the same theoretical framework adopted by FFCRB90. We have employed Eqs. (2) and (6) in FFCRB90 for the magnitudes of the bump and the ZAHB at $\log T_{\text {eff }}=3.85$. Recall that there is evidence that Eq. (2) in FFCRB90 yields too bright bump magnitudes, so that the $\Delta M_{V \text { bump }}^{\mathrm{HB}} \equiv M_{V}^{\mathrm{bump}}-M_{V}^{\mathrm{HB}}$ values thus derived should not be directly compared with observed ones.

The helium and $\alpha$-elements abundances have been drawn from the chemical evolution models discussed in the previous section (cf. Table 1 and Fig. 5).

Values of $\delta \Delta V_{\text {bump }}^{\mathrm{HB}}$ (where $\delta \equiv \mathrm{MRGC1}$ - MRGC2) computed in this way can be found in Table 2. The different entries correspond to different absolute ages assumed for MRGC1 (column 1) and to different age ratios (column 2) between MRGC1 and MRGC2, as obtained from Table 1.

Inspection of this table discloses that the bump in MRGCs with $[\mathrm{Fe} / \mathrm{H}] \simeq-0.2$ should, according to the models, be located at a fainter magnitude level (with respect to the cluster's HB) in comparison with that in a MRGC like 47 Tuc, if Case 1 or Case 2 models are appropriate for MRGC2. Case 3 models may imply somewhat brighter bumps in such clusters. FFCRB90 determine, for 47 Tuc, $\Delta V_{\text {bump }}^{\mathrm{HB}} \simeq 0.45 \pm 0.16 \mathrm{mag}$; Ferraro et al. (1994) have found $\Delta V_{\text {bump }}^{\mathrm{HB}} \simeq 0.52 \pm 0.07 \mathrm{mag}$ for the similarly metal-rich M69. These values may be used with the data in Table 2 to estimate the actual expected location of the bump in MRGC2. $V^{\mathrm{HB}}$ here refers to the lower envelope of the HB distribution.

The employed expressions for the magnitudes of the bump and the HB at the RR Lyrae level have been obtained from models which employ old input physics - in particular, old opacity tables. Both the Straniero \& Chieffi (1991) models, for evolution prior to the HB, and the CCP91 models, for evolution on the HB, represent a significant improvement in this respect, since they incorporate more recent opacities in comparison with those upon which the FFCRB90 analysis is based. 
As a consequence of this difference in the input physics, the updated bump and HB models become progressively fainter than the older models for $Z>10^{-3}$. They do agree quite well, however, at lower metallicities, so that the conclusions by FFCRB90 for their metal-poorest clusters should not be altered appreciably. For the metal-rich objects, on the other hand, a possible correction term must be estimated.

This has been accomplished on the basis of Straniero \& Chieffi's (1991) expression for the magnitude of the bump as a function of age and metallicity and the present synthetic clump models based upon the CCP91 evolutionary tracks. Unfortunately, however, both studies are restricted to $Y_{\mathrm{MS}}=0.23$, so that we cannot analyze the direct impact of the new opacities upon the results displayed in Table 2, which are based upon our chemical evolution models. Also, the bump analysis of Straniero \& Chieffi is strictly valid only for $Z \leq 6 \times 10^{-3}$, so that extrapolation toward higher metallicities is needed.

Adopting $Z=0.006$ for MRGC1 and $Z=0.02$ for MRGC2, we find that improved bump predictions for these objects become fainter than the earlier ones by $0.12 \mathrm{mag}$ and $0.43 \mathrm{mag}$, respectively. This result is only weakly dependent on the assumed age for the clusters. For the HB, on the other hand, the net change amounts to $\approx 0.98-0.82=0.16 \mathrm{mag}$ and $\approx 1.33-0.86=0.47$ mag, respectively. Since these values essentially cancel out in the computation of $\Delta M_{V} \mathrm{HB}$ bump , the results given in Table 2 may be considered as actual standard predictions of the bump location in MRGCs. If observed, deviations from these results should have important implications for our understanding of the internal processes taking place in red giant stars. The reader should bear in mind, however, that more extensive models for several different chemical composition combinations are needed to put these figures on a firmer basis.

A related question that may be raised concerning RGB bumps is: how numerous are the bump stars expected to be in a MRGC? King et al. (1985), Castellani et al. (1989) and FFCRB90 have all pointed out that the probability of detection of the RGB bump increases with increasing metallicity, so that bump stars may be relatively numerous in MRGCs.

To answer this question, one may make use of the theoretical relations which have been obtained by Buzzoni et al. (1983). An illustrative example may be given for the case $t_{9}=10$, $Y_{\mathrm{MS}}=0.28, Z=0.02$. We apply a correction factor to the bump lifetime estimate given by Buzzoni et al. in their Eq. (5b), given that such an estimate apparently refers only to the phase where the luminosity drops, whereas observationally what is of interest is the full region on the RGB where the star crosses the same luminosity levels more than once. A factor of 2.5 has been estimated, on the basis of a comparison of the Bono \& Castellani (1992) and Castellani \& Castellani (1993) diagrams with the predictions by Buzzoni et al. for the same chemical composition and masses. Adopting an overall mass loss on the RGB $\Delta M=0.15 M_{\odot}$, we find $t_{\text {bump }} / t_{\text {clump }} \approx 2.5 \times 0.08=0.2$. This result is essentially insensitive to the assumed amount of mass loss, given that the HB lifetime is only weakly dependent on the stellar mass. The following trends are present, though: decreasing the helium abundance by $\Delta Y=0.05$, we have 
$t_{\text {bump }} / t_{\text {clump }} \approx 0.31$; decreasing the metallicity by $\Delta Z=0.01$, we obtain $t_{\text {bump }} / t_{\text {clump }} \approx 0.14$;

increasing the age by $\Delta t_{9}=5$, we find $t_{\text {bump }} / t_{\text {clump }} \approx 0.16$. To summarize, roughly $20 \%$ as many bump stars as clump objects should be present in MRGCs, with the precise amount depending on the chemical composition and age. For comparison, a similar exercise assuming the Case 1 composition (cf. Table 1 ) to be valid suggests $t_{\text {bump }} / t_{\text {clump }} \approx 2.5 \times 0.044=0.11$ for 47 Tuc, for an age $t_{9}=14$. To be sure, these figures too may depend on the assumptions about mixing at the bottom of the convective layer (cf. Fig. 2 in Bono \& Castellani 1992).

However, since RGB bumps have not been unequivocally identified in the MRGCs observed by Ortolani, Barbuy \& Bica (1994 and references cited therein), the comparison between the above predictions and the observations must be postponed until the HST diagrams for these and other metal-rich objects become available. The latter should represent an important test of our theories of the evolution of red giants and the underlying input physics, and it is our hope that the above summary will prove useful in future investigations of these data.

\section{Summary and discussion}

One of the purposes of the present paper was to discuss the morphological characteristics of the clump of stars in the CMDs of MRGCs which has been associated with the core helium-burning phase (the HB). With this purpose, synthetic HB models for metal-rich compositions, with and without differential reddening, have been constructed and discussed.

We find that few combinations of chemical composition parameters and evolutionary masses on the HB may lead to tilted synthetic HB models. For high $Y$ values, some of the models do appear sloped in the $M_{V}, B-V$ diagram due to blanketing, and we expect that, for $Y_{\mathrm{MS}}>0.3$, tilted structures would become more common as a natural consequence of the properties of the HB evolutionary tracks for high $Y$. In these models, the blueward loops of stars evolving away from the red ZAHB are characterized by a slope $\mathrm{d} \log L / \mathrm{d} \log T_{\text {eff }}>0$ - the slope increasing with increasing $Y$. These trends could not be pursued any further in terms of synthetic calculations in the present study, due to the lack of sufficiently extensive grids of evolutionary tracks for high $Y$. We emphasize the need of synthetic calculations employing extensive grids (in $Y, Z$, and $M_{\mathrm{HB}}$ ) of HB evolutionary tracks to perform more detailed comparisons with the observed CMDs, since neither ZAHB sequences nor individual evolutionary tracks are expected to be able to adequately represent the observed structures (Dorman et al. 1989).

The much more common "clumpy" structures that we find may become tilted due to the effect of differential reddening in the field of a MRGC, although the computations suggest that a large amount of differential reddening should be present in order to induce a noticeably sloped HB.

The age differences between two MRGCs are found to depend sensitively on the chemical evolution scenario adopted for these objects, an important point to bear in mind in future applications of the $\Delta V$ method, especially since disk and bulge globulars may not have shared a 
common chemical enrichment history. Indeed, disk and bulge globulars of similar $\Delta V$ and $[\mathrm{Fe} / \mathrm{H}]$ may not have the same ages, if their $Y$ and $[\alpha / \mathrm{Fe}]$ are appreciably different. In particular, for a given $\Delta V$ and $[\mathrm{Fe} / \mathrm{H}]$, a cluster which was formed from material enriched predominantly by SN II ejecta will appear younger than another one which also suffered enrichment from SN Ia explosions. Depending on the chemical evolution scenario adopted for the bulge, our analysis shows that the bulge globulars may be younger or older than a cluster like 47 Tuc. We believe that this should serve as an important motivation for observers to determine the abundances of the $\alpha$-elements and of helium in MRGCs.

Zinn $(1990,1991)$ has argued that there may not be a genuine bulge system of GCs in the Galaxy. Minniti's (1995b) more recent analysis, on the other hand, suggests that at least some of the MRGCs may belong to the bulge. More critical work appears needed to classify the individual MRGCs into disk and bulge subsystems: our analysis demonstrates that the possibly different chemical evolution histories of the disk and bulge globulars will have to be seriously considered in more quantitative analyses of their CMD properties.

The predicted location of the RGB bump has also been critically discussed in the present work. We provide magnitude estimates for the bump in a MRGC with $[\mathrm{Fe} / \mathrm{H}]=-0.2$ for different chemical evolution scenarios. The RGB bump is expected to be roughly $20 \%$ as well populated as the helium-burning clump in the same cluster.

The authors wish to thank Drs. S. Ortolani, B. Barbuy, and E. Bica for useful discussions; they are especially grateful as well to Dr. B. Carney for his careful reading of a draft of this paper, and for providing several relevant comments and suggestions. M. C. would like to thank Dr. Ben Dorman for useful discussions on the subject of model atmospheres computations, and J. C. Correia and F. G. Garcia for their help in obtaining one key reference. Financial support by FAPESP is acknowledged (grant 92/2747-8). 


\section{REFERENCES}

Alongi, M., Bertelli, G., Bressan, A., and Chiosi, C. 1991, A\&A, 244, 95

Armandroff, T. E. 1988, AJ, 96, 588

Arnett W. D., 1990, in Chemical and Dynamical Evolution of Galaxies, eds. F. Ferrini, J. Franco and F. Matteucci (ETS Editrice, Pisa), p. 410

Barbuy, B., de Freitas Pacheco, J. A., and Castro, S. 1994, A\&A, 283, 32

Beers, T. C., and Sommer-Larsen, J. 1995, ApJS, 96, 175

Bell, R. A. 1992, MNRAS, 257, 423

Bell, R. A., and Gustafsson, B. 1978, A\&AS, 34, 229

Bell, R. A., and Gustafsson, B. 1989, MNRAS, 236, 653

Bell, R. A., and Tripicco, M. 1995, in preparation

Bolte, M. 1992, PASP, 104, 794

Bono, G., and Castellani, V. 1992, A\&A, 258, 385

Brown, J. A., and Wallerstein, G. 1992, AJ, 104, 1818

Brown, J. A., Wallerstein, G., and Oke, J. B. 1990, AJ, 100, 1561

Buzzoni, A., Fusi Pecci, F., Buonanno, R., and Corsi, C. E. 1983, A\&A, 128, 94

Caputo, F., Martinez Roger, C., and Paez, E. 1987, A\&A, 183, 228

Carney, B. W., Storm, J., and Jones, R. V. 1992, ApJ, 386, 663

Carney, B. W., Storm, J., and Williams, C. 1993, PASP, 105, 294

Castellani, M., and Castellani, V. 1993, ApJ, 407, 649

Castellani, V., Chieffi, A., and Norci, L. 1989, A\&A, 216, 62

Castellani, V., Chieffi, A., and Pulone, L. 1991, ApJS, 76, 911 (CCP91)

Castellani, V., Chieffi, A., and Straniero, O. 1992, ApJS, 78, 517

Catelan, M. 1992, A\&A, 261, 443

Catelan, M. 1993, A\&AS, 98, 547

Catelan, M., and de Freitas Pacheco, J. A. 1995, A\&A, 297, 345

Catelan, M., de Freitas Pacheco, J. A., and Horvath, J. E. 1995, ApJ, in press

Chieffi, A., Straniero, O., and Salaris, M. 1991, in The Formation and Evolution of Star Clusters, Astronomical Society of the Pacific Conference Series 13, ed. K. Janes (San Francisco, ASP), p. 219

Davidge, T. J., and Simons, D. A. 1994, AJ, 107, 240

Demarque, P., and Lee, Y.-W. 1992, A\&A, 265, 40 
Dorman, B. 1992, ApJS, 81, 221

Dorman, B. 1994, private communication

Dorman, B., VandenBerg, D. A., and Laskarides, P. G. 1989, ApJ, 343, 750

Fernley, J. 1993, A\&A, 268, 591

Ferraro, F. R. 1992, MSAIt, 63, 491

Ferraro, F. R., Fusi Pecci, F., Guarnieri, M. D., Moneti, A., Origlia, L., and Testa, V. 1994, MNRAS, 266, 829

de Freitas Pacheco, J. A. 1993, ApJ, 403, 673

Fusi Pecci, F., Ferraro, F. R., Crocker, D. A., Rood, R. T., and Buonanno, R. 1990, A\&A, 238, 95 (FFCRB90)

Guarnieri, M. D., Montegriffo, P., Ortolani, S., Moneti, A., Barbuy, B., and Bica, E. 1995, The Messenger, 79, 26

Hesser, J. E., Harris, W. E., VandenBerg, D. A., Allwright, J. W. B., Shott, P., and Stetson, P. B. 1987, PASP, 99, 739

Hill, G. 1982, Publ. Dom. Astrophys. Obs., 16, 67

Iben, I. Jr. 1968, Nature, 220, 143

Iben, I. Jr., and Rood, R. T. 1970, ApJ, 161, 587

Idiart, T. E. P., de Freitas Pacheco, J. A., and Costa, R. D. D. 1995, AJ, in press

King, C. R., Da Costa, G. S., and Demarque, P. 1985, ApJ, 299, 674

Kurucz, R. L. 1979, ApJS, 40, 1

Kurucz, R. L. 1991, in Stellar Atmospheres, ed. A. G. Davis Philip, D. S. Hayes and J. W. Liebert (L. Davis Press, Schenectady), p. 129

Kurucz, R. L. 1992, in The Stellar Populations of Galaxies, Proc. IAU Symp. 149, eds. B. Barbuy and A. Renzini (Kluwer, Dordrecht), p. 225

Majewski, S. R. 1993, ARA\&A, 31, 575

Marquez, A., and Schuster, W. J. 1994, A\&AS, 108, 341

Matteucci, F. 1995, in Unsolved Problems in the Milky Way, Proc. IAU Symp. 169, ed. L. Blitz (Kluwer, Dordrecht), in press

McWilliam, A., and Rich, R. M. 1994, ApJS, 91, 749

Minniti, D. 1995a, A\&A, 300, 109

Minniti, D. 1995b, AJ, 109, 1663

Nomoto, K., Thielemann, F.-K., and Yokoi, K. 1984, ApJ, 286, 644

Ortolani, S. 1994, private communication 
Ortolani, S., Barbuy, B., and Bica, E. 1990, A\&A, 236, 362 (OBB90)

Ortolani, S., Barbuy, B., and Bica, E. 1994, A\&AS, 108, 653

Ortolani, S., Bica, E., and Barbuy, B. 1992, A\&AS, 92, 441

Ortolani, S., Bica, E., and Barbuy, B. 1993, A\&A, 267, 66

Ratag, M. A., Pottasch, R. S., Dennenfeld, M., and Menzies, J. W. 1992, A\&A, 255, 255

Renzini, A. 1991, in Observational Tests of Cosmological Inflation, eds. T. Shanks, A. J. Banday, R. S. Ellis, C. S. Frenk and W. Wolfendale (Kluwer, Dordrecht), p. 131

Renzini, A. 1994, A\&A, 285, L5

Renzini, A., and Greggio, L. 1990, in Bulges of Galaxies, Proc. ESO/CTIO Workshop, eds. B. J. Jarvis and D. M. Terndrup (ESO, Garching), p. 47

Salaris, M., Chieffi, A., and Straniero, O. 1993, ApJ, 414, 580

Salpeter, E. E. 1955, ApJ, 121, 161

Sandage, A. 1993, AJ, 106, 703

Sandage, A., and Cacciari, C. 1990, ApJ, 350, 645

Seidel, E., Demarque, P., and Weinberg, D. 1987, ApJS, 63, 917

Skillen, I., Fernley, J. A., Stobie, R. S., and Jameson, R. F. 1993, MNRAS, 265, 301

Straniero, O., and Chieffi, A. 1991, ApJS, 76, 525

Sweigart, A. V. 1987, ApJS, 65, 95 (SW87)

Sweigart, A. V., and Gross, P. G. 1976, ApJS, 32, 367

Terndrup, D. M., and Walker, A. R. 1994, AJ, 107, 1786

Tripicco, M. J., Dorman, B., and Bell, R. A. 1993, AJ, 106, 618

van den Bergh, S. 1991, Phys. Rep., 204, 386

VandenBerg, D. A. 1992, ApJ, 391, 685 (VdB92)

VandenBerg, D. A., and Bell, R. A. 1985, ApJS, 58, 561

VandenBerg, D. A., Bolte, M., and Stetson, P. B. 1990, AJ, 100, 445

Zinn, R. 1985, ApJ, 293, 424

Zinn, R. 1990, JRASC, 84, 89

Zinn, R. 1991, in The Formation and Evolution of Star Clusters, Astronomical Society of the Pacific Conference Series 13, ed. K. Janes (San Francisco, ASP), p. 532 
Fig. 1. - Synthetic clump models in the $M_{V}, B-V$ diagram. The basic assumptions made in the calculations are summarized in the upper left portions of the panels.

Fig. 2.- In panel a, a clumpy synthetic HB model, similar to those depicted in Fig. 1, is displayed. In panels c through d, increasing amounts of (uniform) differential "reddening" are assumed to be present in the field of the cluster: $\Delta E(B-V)=0.06,0.10$, and 0.15 mag, respectively. Besides increasing the dispersion in the diagrams, differential reddening clearly works in the sense of producing sloped structures: formal least-squares solutions with $B-V$ as the independent variable give $\mathrm{d} V / \mathrm{d}(B-V) \simeq 0.02,0.33,0.74$, and 1.26 for the models displayed in panels a through $\mathrm{d}$, respectively.

Fig. 3.- As in Figs. 2a and b, except that a model already presenting a significant slope in the observational diagram without differential reddening having been included (panel a) is shown. The formal least-squares slope values in panels a and $\mathrm{b}$ are $\mathrm{d} V / \mathrm{d}(B-V) \simeq 0.69$ and 0.80 , respectively.

Fig. 4.- Synthetic clump model equivalent to Fig. 3a in the $M_{\text {bol }}-\log T_{\text {eff }}$ diagram. To be noted is that the blanketing effect is largely responsible for the production of a significant tilt in the CMD of Fig. 3a.

Fig. 5.- The helium abundance by mass $Y$ (panel a) and oxygen-to-iron ratio $[\mathrm{O} / \mathrm{Fe}]$ (panel b) as a function of the metallicity $[\mathrm{Fe} / \mathrm{H}]$, as predicted by three different models for the chemical evolution of the Galactic bulge. See text for more details. 